\title{
Oral health promotion initiated during pregnancy successful in reducing early childhood caries
}

\author{
Abstracted from \\ Plutzer K, Spencer AJ. \\ Efficacy of an oral health promotion intervention in the prevention of early childhood caries. \\ Community Dent Oral Epidemiol 2008; 36: 335-346 \\ Address for correspondence: Kamila Plutzer, Australian Research Centre for Population Oral Health, \\ School of Dentistry, Faculty of Health Science, University of Adelaide, Adelaide, SA 5005, Australia. \\ E-mail: kamila.plutzer@adelaide.edu.au
}

\section{Question: Can an oral health promotion programme starting during pregnancy prevent early childhood caries?}

Design This was a randomised controlled trial using Zelen's design. ${ }^{1}$ Intervention Women in the test group received three rounds of printed information supplied in the form of anticipatory guidance. The first round of information was provided at enrolment. The second and the third rounds of information were mailed to the mother's home address when their child reached 6 and 12 months of age. The topics covered in each round offered mothers information and support relevant to their own and the child's oral development across the subsequent 6 months period. In addition, the first round of literature included information for expectant mothers related to oral health changes during pregnancy, with emphasis on oral hygiene during pregnancy and proper nutrition. Other topics covered in this round were the importance of primary teeth, the use of pacifiers and the sleeping patterns of the child. The second and third rounds of information were concerned with the eruption of teeth, oral hygiene and nutrition. The oral health promotion material was supplemented with the existing nutritional recommendations. Half of the mothers in the test group were randomly selected to test the impact of a structured telephone consultation as an additional mode of contact. The structured telephone consultation was conducted as a scripted interview. Four questionnaires were also conducted (a maternal oral health survey, refusal to participate, child's oral health survey, and evaluation of oral health intervention). Outcome measures The main outcome was severe early childhood caries (S-ECC). A case of S-ECC was defined when one or more upper incisor teeth labial surfaces were carious, either noncavitated or cavitated. Diagnosis was based on visual criteria only. Results Out of 649 women enrolled in the programme (test group, 327 control group, 322), 441 had their child examined at followup. The incidence of S-ECC in the test group was $1.7 \%$ and in the control group was $9.6 \%(P<0.001)$.

Conclusions An oral health promotion programme based on repeated rounds of anticipatory guidance initiated during the mother's pregnancy was successful in reducing the incidence of S-ECC in these very young children.

\section{Commentary}

Oral health promotion has traditionally suffered from a lack of highquality evaluation to support its effectiveness in improving oral health. ${ }^{2}$ In this study, the authors used a robust research method to evaluate the efficacy of anticipatory guidance in preventing severe early childhood caries (S-ECC). The study has a clearly focused question with a specific population, intervention and outcome measure.

The recently published review of the National Health Service (NHS) dental services in England (www.dh.gov.uk/en/ Publicationsandstatistics/Publications/

PublicationsPolicyAndGuidance/DH_101137) has re-affirmed the need for a preventive focus in the delivery of NHS services. Current health policy focuses on a preventative approach, particularly in antenatal care and for the early years. (www.scotland.gov.uk/ Publications/2007/12/11103453/0) This trial is therefore particularly relevant for the professionals who are involved in improving oral health of children and pregnant mothers.

An unusual feature of this trial is the use of Zelen's design, which involves randomisation prior to consent to participate. This confers a number of advantages, namely, enhanced recruitment, a reduction in resentful demoralisation in participants and a reduced Hawthorne effect in the control group. ${ }^{3}$ Lack of blinding may introduce bias, however. ${ }^{4}$ The authors highlight the potential loss of statistical power if participants refuse the allocated group, but the rate of transfer between groups in this study was low, at $0.8 \%$. The main advantage cited here was a practical one in that the intervention could begin immediately once the consent form was signed. Randomisation was appropriately carried out using a random number table, with subsequent analysis of baseline characteristics revealing both groups to be well matched with even distribution of potentially important confounders.

Participants are all accounted for in a flow diagram in accordance with the CONSORT guidelines. ${ }^{5}$ Exclusions are clearly specified and all losses are reported, which is commendable. Unfortunately, it is not clear if the authors performed an intention to treat analysis, which should be undertaken when using a Zelen design because of the potential for movement between control and test groups, which may have had a bearing on the final results. The sample size is justified with some clear power calculations, and it is disappointing that the final numbers in both the test and control groups fell slightly short of the mark.

The main results are presented in an easy-to-follow table and demonstrate a statistically significant reduction in incidence of S-ECC in the test group, with respect to the provision of anticipatory 
guidance. The telephone consultation with mothers did not influence the incidence of S-ECC, but this could have been because of an insufficient sample size in this section of the trial. Further analysis to control for heterogeneity between the groups demonstrated that age of child at examination and familial structure were independent variables for prediction of S-ECC development. Following adjustment for these potentially confounding variables the effect of the intervention remained.

The authors themselves identified three main limitations which may hinder its ready application. First, it is not known how many noncavitated lesions will progress to cavitation. Second, there was no test-retest reliability. This is particularly important given the subjective nature of recording noncavitated lesions. Finally, the examiner was not masked to study-group allocation which may have led to detection bias. In addition, the examiner calibration is ambiguous.

In terms of application in a local setting, this intervention could easily be implemented and appears to be successful in reducing S-ECC incidence in preschool children, notwithstanding the limitations outlined above. A number-needed-to-treat of 13 was calculated for this study, indicating that, from a financial point of view, provision of printed information in the form of anticipatory guidance may be a cost-effective approach in improving the oral health of young children. Overall, the results are encouraging for those involved in oral health promotion aimed at pregnant mothers and young children. For example, delivery of anticipatory oral health messages from a young age is one of the elements of the ongoing Childsmile programme (www.child-smile.org).

Siobhan M Lucey

Department of Public Health, NHS Lothian, Edinburgh,

Scotland, UK

1. Torgerson DJ, Roland M. What is Zelen's design? BMJ. 1998; 316: 606.

2. Kay E, Locker D. A systematic review of the effectiveness of health promotion aimed at improving oral health. Community Dent Health 1998; 15: 132-144.

3. Adamson J, Cockayne S, Puffer, Torgerson DJ. Review of randomised trials using the post-randomised consent (Zelen's) design. Contemp Clin Trials 2006; 27: 305-319.

4. Day SJ, Altman DG. Blinding in clinical trials and other studies. Br Med J 2000; 321: 504

5. Altman DG, Schulz KF, Moher D, et al. The revised CONSORT statement for reporting randomized trials: explanation and elaboration. Ann Intern Med 2001; 134: 663-694.

Evidence-Based Dentistry (2009) 10, 100-101. doi:10.1038/sj.ebd.6400677 